Chl or ophyl I Fl uor escence Neasur ement s in Ar abi dopsi s Pl ant s Usi ng a Pul se- ampl i t ude- modul at ed (PAM Fl uor omet er

\begin{tabular}{|l|l|}
\hline 著者 & Not ohashi Rei ko, Myouga Fum yoshi \\
\hline $\begin{array}{l}\text { j our nal or } \\
\text { publ i cat i on ti t l e }\end{array}$ & Bi o- pr ot ocol \\
\hline vol une & 5 \\
\hline number & 9 \\
\hline page range & e1464 \\
\hline year & $2015-05-05$ \\
\hline 出版者 & LLC \\
\hline 権利 & ( C) 2015 Bi o- pr ot ocol LLC. \\
\hline URL & ht t p: //hdl . handl e. net /10297/9164 \\
\hline
\end{tabular}


Category by field: Plant Science $>$ Plant physiology $>$ Photosynthesis

2 Category by field: Plant Science $>$ Plant biochemistry $>$ Other compound

3 Category by field: Biochemistry $>$ Other compound $>$ Chlorophyll

4 Category by organism: Plants $>$ Arabidopsis $>$ Leaf $>$ Other compound

\section{Chlorophyll Fluorescence Measurements in Arabidopsis plants using a} pulse-amplitude-modulated (PAM) fluorometer

Reiko Motohashi* and Fumiyoshi Myouga ${ }^{1}$

Shizuoka University, Agriculture department, Shizuoka city, Shizuoka, Japan

1.RIKEN Center for Sustainable Resource Science, 1-7-22 Suehirocho, Tsurumi-ku, Yokohama, Kanagawa 230-0045, Japan

*For correspondence: armotoh@ipc.shizuoka.ac.jp

[Abstract] In this protocol, to analyze PSII activity in photosynthesis, we measure the $\mathrm{Fv} / \mathrm{Fm}$ $(\mathrm{Fv}=\mathrm{Fm} \pm \mathrm{Fo}$ ) value ( $\mathrm{Fo}$ and $\mathrm{Fm}$ are the minimum and maximum values of chlorophyll fluorescence of dark-adapted leaves, respectively). Fv/Fm is a reliable marker of photo- inhibition (Krause et al., 1988). Chlorophyll fluorescence in leaves was measured at room temperature using a photosynthesis yield analyzer (MINI- PAM, Walz, Effeltrich, Germany) and a pulse-amplitude-modulated (PAM) fluorometer (TEACHING-PAM, Walz, Effeltrich, Germany).

\section{Materials and Reagents}

1. Arabidopsis plants Note: We plated Nossen ecotype seeds that had been surface-sterilized on germination medium (GM) agar plates (Motohashi et al., 2003) containing 1\% sucrose, with the appropriate selection agent (antibiotic or herbicide) per specific genotype. Plants were kept at $4{ }^{\circ} \mathrm{C}$ for 3 days to improve germination rates and then grown in lighted growth chambers (CF-405, TOMY-Seiko, Tokyo, Japan) with approximately $75 \mu \mathrm{mol}$ photon $/ \mathrm{m}^{2} / \mathrm{s}$ at $22{ }^{\circ} \mathrm{C}$ under a $16 \mathrm{~h}$-light /8-h dark cycle (long-day conditions) for 3 weeks.

\section{Equipment}

1. Photosynthesis yield analyzer (MINI- PAM) (the equipment used in this protocol) (Figure 1). Compact design and easy operation are the most outstanding features of the 
MINI-PAM. This device is in particular well-suited for determination of quantum yield and

\section{Procedure}

Protocol for using the MINI-PAM as referenced from the official instruction manual (TEACHING-PAM has a similar protocol and as such is not included here). Basically the most relevant fluorescence parameters of MINI-PAM are automatically obtained by a single key operation within a second and up to 4,000 data sets are stored for future analysis:

(For reference, information regarding the MINI-PAM-II can be downloaded from the following URL: http://www.walz.com/downloads/manuals/mini-pam-II/MINI-PAM-II Broschure.pdf)

1. Plants grown normally for 3 weeks are dark-adapted for $20 \mathrm{~min}$ before chlorophyll fluorescence measurements. *In our case, dark-adapted means the plants are kept either in a dark drawer (for plated plants) or covered with a large box (for potted plants), in both cases in rooms with dark curtains and no artificial light sources.

2. Setup the MINI-PAM components. Additional peripheral components were connected to the four sockets at the side of the MINI-PAM Main Control Unit. PIN-assignments of "LEAF CLIP", "RS 232", "OUTPUT" and "CHARGE" indicate a Leaf Clip holder 2030-B, Computer control, Chart recorder and Battery Charger, respectively. The MINI-PAM was conceived 
as a typical stand-alone instrument for field experiments. Thus the actual measurement of the most relevant YIELD-parameter (quantum yield of photochemical energy conversion) just connected the fiberoprtics and leaf clip holder without conjunction with a PC and the WinControl software. So this protocol introduces the basic operation of the MINI-PAM without using computer control.

3. Activate the MINI-PAM by pressing the "ON" button. Under standard conditions, the measuring light is on automatically.

4. The AUTO-ZERO function (MODE-menu point 2) should be applied to determine the signal in absence of sample (background signal). To move to MODE-menu point 2, press "MODE" button (possible to omit) and " $\wedge$ " button one times to select 2 of 51 points of the MODE-menu. Then push "SET" button to set the F value to zero (not stable, blinking) on measuring light (Figure 2).

5. Place a dark-adapted leaf sample on the measuring head of the Leaf Clip holder. The distance between sample and fiberoptics should be about 10-15 mm (Figure 3). We dark-adapt the plants by either putting them in drawers (for dished plants) or covering them with boxes (for potted plants) - in both cases dark curtains are used and all artificial lights are turned off. Temperature when measuring should be the same as the growth environment.

6. Just press the "START" button. Measuring the fluorescence parameters is proceeding automatically within seconds (see below).

(1) the minimum fluorescence in dark-adapted state (Fo) is sampled (displayed as ...F).

(2) a saturation pulse is applied.

(3) a saturation pulse induced maximum fluorescence in dark-adapted state $(\mathrm{Fm})$ is sampled (displayed as ....M).

(4) $\mathrm{YIELD}=(\mathrm{Fm}-\mathrm{Fo}) / \mathrm{Fm}=\mathrm{Fv} / \mathrm{Fm}$ is calculated and shown on the display as ...Y.

(5) When you use the Leaf Clip holder, the photosynthetically active radiation (PAR) and temperature at the same spot of a leaf where fluorescence is measured is also sampled (displayed as ...L and ...C, respectively).

(6) the apparent rate of electron transport $(E T R)=$ YIELD $\times$ PAR $\times 0.5 \times$ ETR-factor (0.84) is calculated (displayed as ...E).

7. The parameter indicated by the above is shown to a screen after measurement (Figure 4). The obtained data are stored in the MEMORY.

Arabidopsis plants under normal growth condition shows an $\mathrm{Fv} / \mathrm{Fm}$ value between 0.75 to 0.85. (If $\mathrm{Fm} / \mathrm{Fv}$ is not between 0.75 and 0.85 , it is highly likely that the sample Arabidopsis plants are in poor health or not properly grown.) 
On the other hand, when leaf is illuminated, its fluorescence yield can change between Fo

\section{Notes}

1. In order to obtain reliably reproducible data, it is imperative that the plant growth environment be as uniform / consistent as possible. For example depending on light environment the value of a plant chlorophyll fluorescence will fluctuate. The amount of light a plant receives when next to the side light on the growth incubator is completely 
different from the light it receives when on the center of the shelf. For the reason it is important to shuffle the location of growth mediums, etc (Figure 7).

2. At least 5 replicates are measured, with final data being an average of these measurements. As measurement with the MINI-PAM is very easy and results are consistent over each measurement, measuring twice is enough to satisfy technical duplication requirements.

\section{Acknowledgments}

This work was supported by the Ministry of Education, Culture, Sports, Science and Technology (Japan) [Grants-in-Aid for Scientific Research (No.17681022 to R.M.)].

This protocol is modified and appended referencing the original, as featured in Integrated analysis of transcriptome and metabolome of Arabidopsis albino or pale green mutants with disrupted nuclear-encoded chloroplast proteins. Satou M, Enoki H, Oikawa A, Ohta D, Saito K, Hachiya T, Sakakibara H, Kusano M, Fukushima A, Saito K, Kobayashi M, Nagata N, Myouga F, Shinozaki K, Motohashi R. Plant Mol Biol. 2014 Jul;85(4-5):411-28

\section{References}

1. Krause, G. H., Grafflage, S., Rumich-Bayer, S. and Somersalo, S. (1988). Effects of freezing on plant mesophyll cells. Symp Soc Exp Biol 42: 311-327.

2. Motohashi, R., Ito, T., Kobayashi, M., Taji, T., Nagata, N., Asami, T., Yoshida, S., Yamaguchi-Shinozaki, K. and Shinozaki, K. (2003). Functional analysis of the $37 \mathrm{kDa}$ inner envelope membrane polypeptide in chloroplast biogenesis using a Ds-tagged Arabidopsis pale-green mutant. Plant J 34(5): 719-731.

3. Valvekens, D., Van Montagu, M. and Van Lijsebettens, M. (1988) Isolation of genes expressed in specißc tissues of Arabidopsis thaliana by differential screening of a genomic library. Proc. Natl. Acad. Sci. USA, 85, 5536-5540. 


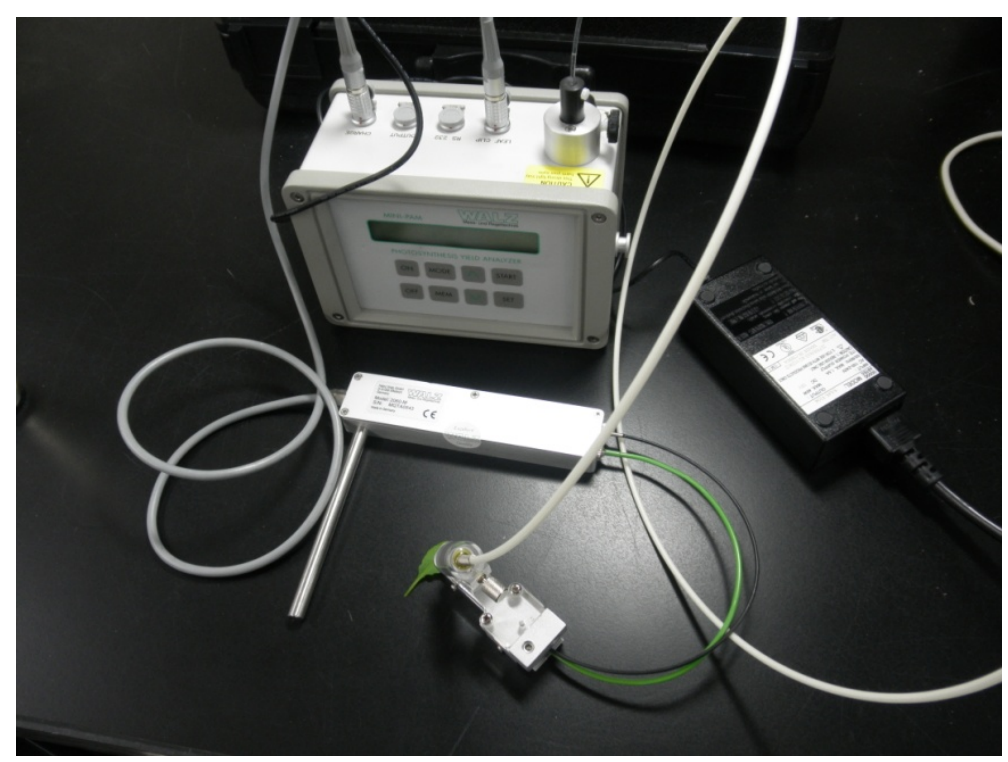

Figure 1. Photosynthesis yield analyzer (MINI-PAM, Walz, Effeltrich, Germany ) 


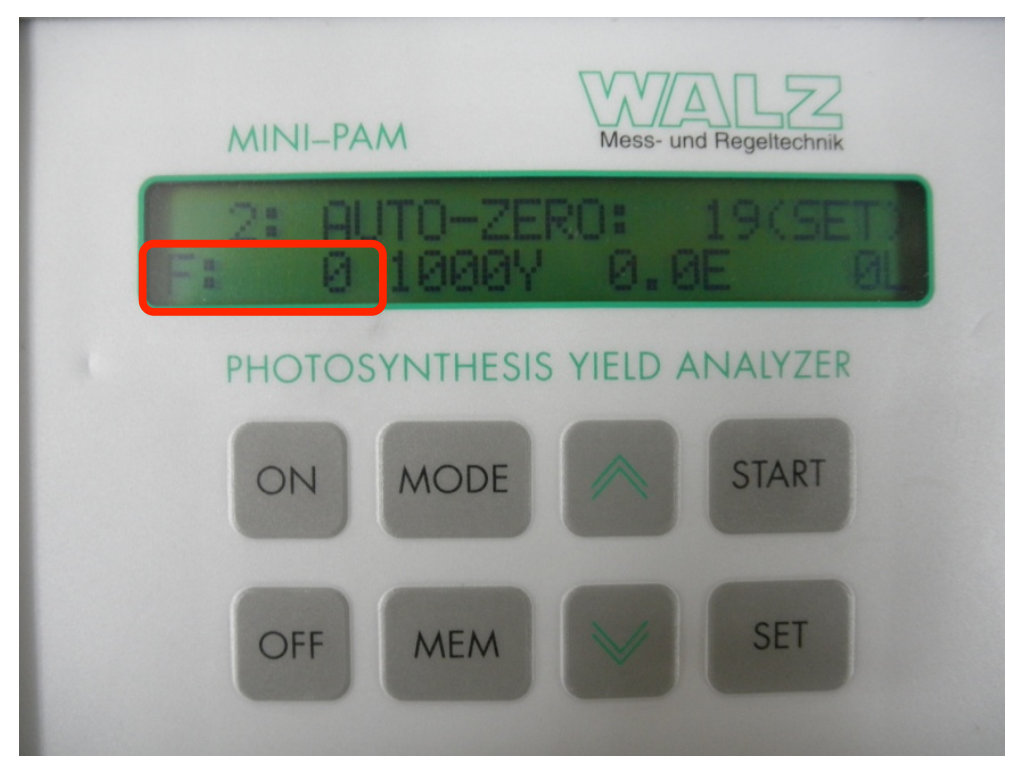

Figure 2. F value blinks " 0 " after pressing the "SET" button. 


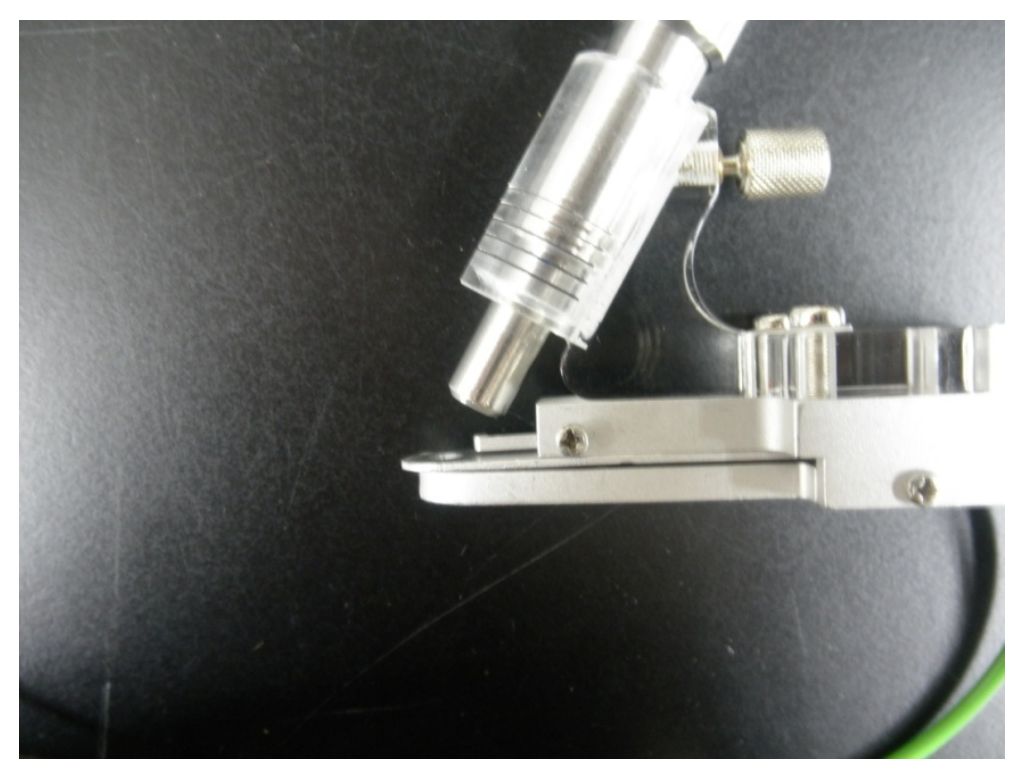

Figure 3. A photo of the leaf clip holder and fiberoptics . 
Figure 4. A photo of the machine display after measurement. 
Figure 5. A photo of the display when setting Fo and Fm ("Mode" menu 25). 
A

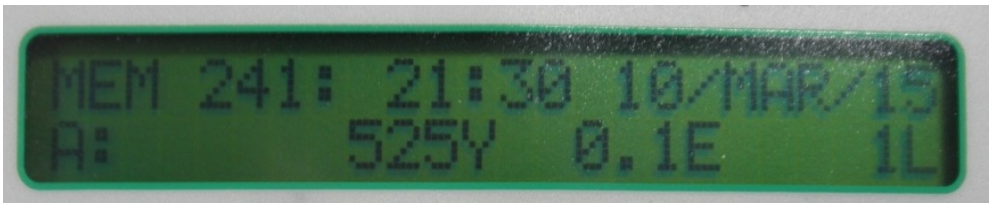

B

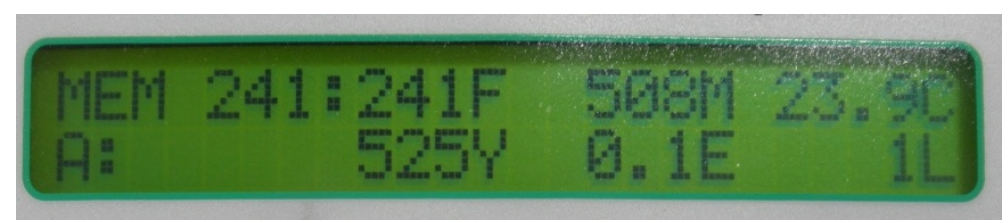

C

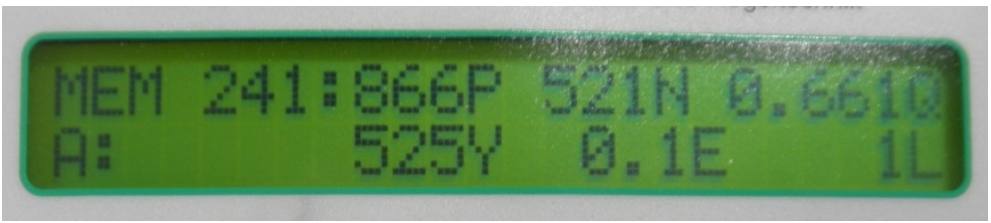

Figure 6. A photo showing the display for "Mode" menu 25.

A. The first display line shows data set number and recording day/ time. The second line shows YIELD (Y), ETR (E) and PAR (L).

$B$. The first display line shows the fluorescence yield measured briefly before saturating light pulse $(F)$, the maximum fluorescence (M) and temperature (C).

C. The first display line shows the quenching coefficients $q P(P), q N$ (N) and NPQ (Q). 


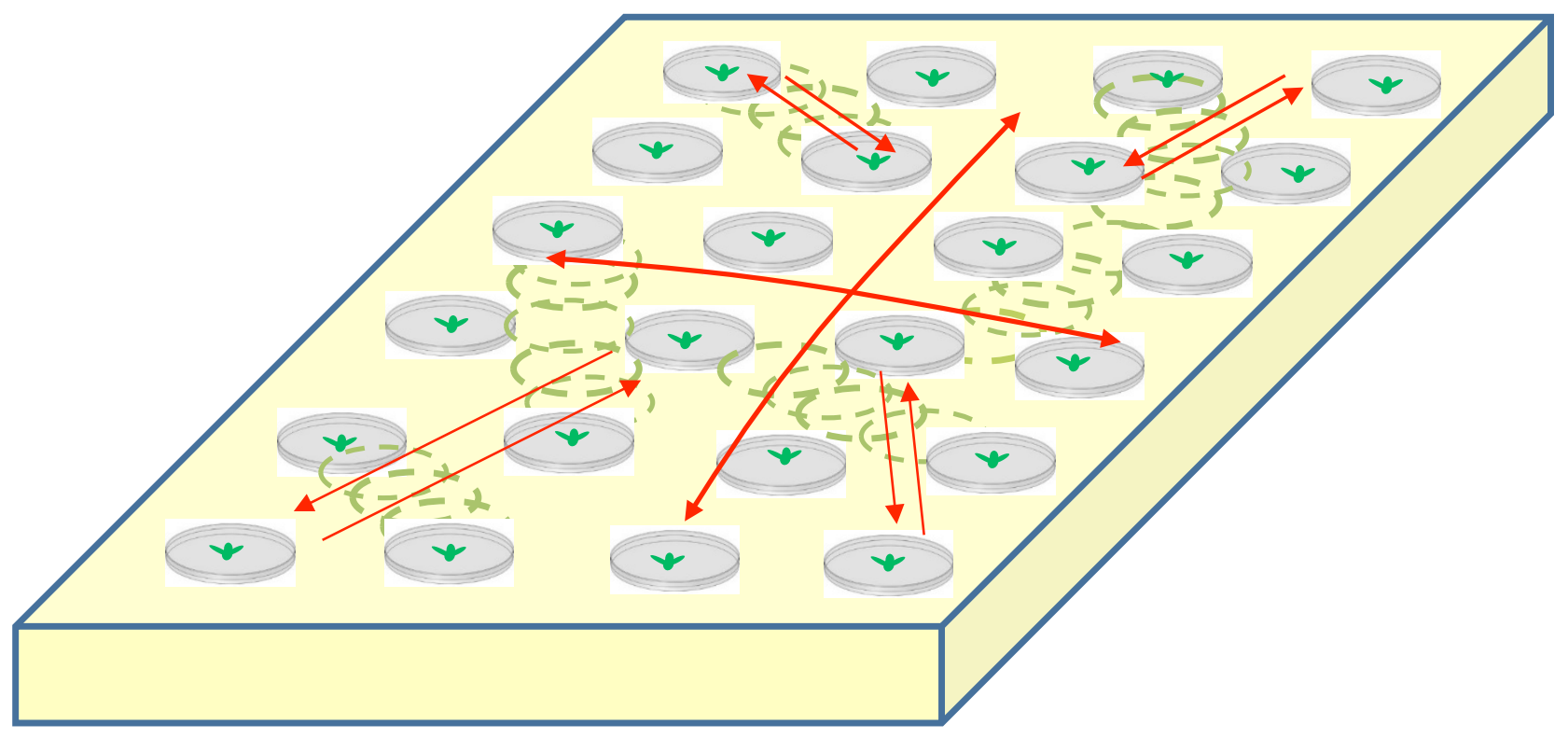

Figure 7. A visual example of how growth mediums might be shuffled. 